OPEN ACCESS

Edited by:

Yong Zhao,

Institute of Zoology (CAS), China

Reviewed by:

Peixiang Lan,

Huazhong University of Science and

Technology, China

$\mathrm{Min} \mathrm{Hu}$,

The University of Sydney, Australia

${ }^{*}$ Correspondence:

Xiaoshun $\mathrm{He}$

gdtrc@163.com

Chenglin Wu

wuchlin7@mail.sysu.edu.cn

Xiaomin Shi

shixm5@mail.sysu.edu.cn

${ }^{t}$ These authors have contributed equally to this work and share first authorship

Specialty section:

This article was submitted to Alloimmunity and Transplantation,

a section of the journal

Frontiers in Immunology

Received: 10 December 2021 Accepted: 24 January 2022

Published: 10 February 2022

Citation:

Tang Y, Wang J, Zhang Y, Li J, Chen M, Gao Y, Dai M, Lin S, He X, Wu $C$ and Shi $X$ (2022) Single-Cell RNA Sequencing Identifies Intra-Graft Population Heterogeneity in Acute Heart Allograft Rejection in Mouse.

Front. Immunol. 13:832573. doi: 10.3389/fimmu.2022.832573

\section{Single-Cell RNA Sequencing} Identifies Intra-Graft Population Heterogeneity in Acute Heart Allograft Rejection in Mouse

\author{
Yunhua Tang ${ }^{1,2,3 \dagger}$, Jiali Wang ${ }^{4 \dagger}$, Yixi Zhang ${ }^{1,2,3 \dagger}$, Jun $\mathrm{Li}^{1,2,3}$, Maogen Chen ${ }^{1,2,3}$, \\ Yifang Gao ${ }^{1,2,3}$, Meiqin Dai ${ }^{1,2,3}$, Shengjie Lin ${ }^{1,2,3}$, Xiaoshun $\mathrm{He}^{1,2,3^{*}}$, Chenglin $\mathrm{Wu}^{1,2,3^{*}}$ \\ and Xiaomin Shi ${ }^{1,2,3 *}$

\begin{abstract}
1 Organ Transplant Center, The First Affiliated Hospital, Sun Yat-sen University, Guangzhou, China, ${ }^{2}$ Guangdong Provincial Key Laboratory of Organ Donation and Transplant Immunology, Guangzhou, China, ${ }^{3}$ Guangdong Provincial International Cooperation Base of Science and Technology (Organ Transplantation), Guangzhou, China, ${ }^{4}$ Department of Nephrology, The First Affiliated Hospital, Sun Yat-sen University, Guangzhou, China
\end{abstract}

Transplant rejection remains a major barrier to graft survival and involves a diversity of cell types. However, the heterogeneity of each cell type in the allograft remains poorly defined. In the present study, we used single-cell RNA sequencing technology to analyze graftinfiltrating cells to describe cell types and states associated with acute rejection in a mouse heart transplant model. Unsupervised clustering analysis revealed 21 distinct cell populations. Macrophages formed five cell clusters: two resident macrophage groups, two infiltrating macrophage groups and one dendritic cell-like monocyte group. Infiltrating macrophages were predominantly from allogeneic grafts. Nevertheless, only one infiltrating macrophage cluster was in an active state with the upregulation of $C D 40$, Fam26f and Pira2, while the other was metabolically silent. Re-clustering of endothelial cells identified five subclusters. Interestingly, one of the endothelial cell populations was almost exclusively from allogeneic grafts. Further analysis of this population showed activation of antigen processing and presentation pathway and upregulation of $\mathrm{MHC}$ class II molecules. In addition, Ubiquitin D was specifically expressed in such endothelial cell population. The upregulation of Ubiquitin $D$ in rejection was validated by staining of mouse heart grafts and human kidney biopsy specimens. Our findings present a comprehensive analysis of intra-graft cell heterogeneity, describe specific macrophage and endothelial cell populations which mediate rejection, and provide a potential predictive biomarker for rejection in the clinic.

Keywords: transplantation, acute rejection, macrophages, endothelial cells, single-cell RNA sequencing 


\section{INTRODUCTION}

Organ transplantation is a preferred choice of treatment for patients with end stage diseases. Despite advances in the field, rejection remains a major barrier to both short-term and longterm graft survival. Currently, immunosuppressive drugs targeting $\mathrm{T}$ cells are widely used in the clinic, which effectively suppresses acute rejection and significantly improves short-term graft survival. Unfortunately, acute rejection still occurs even when $\mathrm{T}$ cells are depleted in some cases, indicating that non- $\mathrm{T}$ cell factors are involved in graft rejection $(1,2)$.

Rejection is a very complex process involving the participation and coordination of all types of immune cells and non-immune cells. Conventional methods, such as microarray and bulk RNA sequencing, have been extensively applied to explore the regulation network associated with rejection $(3,4)$. Our recent work reported a profile of lncRNAs in acute rejection which may provide potential diagnostic markers in the clinic (5). However, in the rejection response, information acquired by traditional assays is incomplete, especially those related to features of each specific cell type. Recently, techniques for single-cell RNA sequencing (scRNA-seq) have been developed and optimized which allow rapid and simultaneous detection of thousands of genes at the single-cell level. scRNA-seq has revolutionized our way to study the complexity of different cell types in an environment $(6,7)$. With scRNA-seq, Wu et al. successfully identified 16 cell types in a single human kidney allograft biopsy and found diverse immune cell infiltrates as well as novel endothelial cell states (8). However, due to limited sample size of kidney biopsy and the high heterogeneity of human samples, the results might be incomplete and skewed. Therefore, we chose to take advantage of scRNA-seq to study acute rejection in the well-established mouse heart transplant model.

In this study, we identified 21 cell populations. As expected, we observed known pathways associated with rejection, such as activation and expansion of $\mathrm{T}$ cells and NK cells. We also characterized heterogeneity of graft infiltrating macrophages. Importantly, we identified a novel endothelial cell subset which can function as antigen presenting cells that potentially mediate transplant rejection. The expression of Ubiquitin D was significantly higher in such endothelial cells, which was confirmed in the mouse allogeneic heart grafts and in the kidney biopsy specimens with rejection.

\section{MATERIALS AND METHODS}

\section{Animals}

Male C57BL/6J and BALB/c mice aged eight to ten weeks were obtained from the Animal Experimental Center of Sun Yat-sen University. All animal care and experiments were approved by the Animal Care Ethics Committee of the First Affiliated Hospital of Sun Yat-sen University.

\section{Heterotopic Cardiac Transplantation}

Mouse heterotopic heart transplantation was performed as described in our previous study (9). Briefly, donor heart grafts were harvested from $\mathrm{C} 57 \mathrm{BL} / 6 \mathrm{~J}$ mice or $\mathrm{BALB} / \mathrm{c}$ mice and then transplanted into the abdominal cavity of recipient C57BL/6J mice via anastomosing the ascending aorta and pulmonary artery of the graft end-to-side to the recipient's aorta and vena cava respectively. Daily transabdominal palpation was used for assessing graft survival, and graft rejection was defined as complete cessation of palpable heartbeats, confirmed by laparotomy. Heart grafts were harvested on Day 5 posttransplant for single-cell transcriptional profiling.

\section{Clinical Samples}

Formalin-fixed, paraffin embedded kidney biopsy specimens were collected from 10 patients who accepted kidney transplantation at the First Affiliated Hospital of Sun Yat-sen University between July 2015 and August 2018. All 10 donors were enrolled in a voluntary organ donation program in China. The study procedure was approved by the Research Ethics Committee of the First Affiliated Hospital of Sun Yat-sen University. Informed consent was obtained from every patient included in the study. The clinicopathologic variables of the patients are as shown in Supplementary Table 1.

\section{Single-Cell Dissociation}

Single-cell RNA-seq experiments were performed in the laboratory of NovelBio Bio-Pharm Technology Co., Ltd. The mouse heart allografts were surgically removed and kept in MACS Tissue Storage Solution (Miltenyi Biotec) until processing. The tissue samples were processed as described below. In brief, samples were first washed with phosphatebuffered saline (PBS), minced into small pieces on ice and enzymatically digested with MACS containing $150 \mathrm{U} / \mathrm{mL}$ collagenase II (Worthington), $275 \mathrm{U} / \mathrm{mg}$ collagenase IV (Worthington), $1.2 \mathrm{U} / \mathrm{mL}$ dispase II (Roche) and $50 \mathrm{U} / \mathrm{mL}$ DNase I (Worthington) for $45 \mathrm{mins}$ at $37^{\circ} \mathrm{C}$ (agitation twice). After digestion, samples were filtered through a $70 \mu \mathrm{m}$ cell strainer, and centrifuged at $300 \mathrm{~g}$ for $5 \mathrm{~min}$. After the supernatant was removed, the pelleted cells were suspended in red blood cell lysis buffer (Miltenyi Biotec) to lyse red blood cells. After washing with PBS containing $0.04 \%$ BSA, the cell pellets were re-suspended in PBS containing 0.04\% BSA and re-filtered through a $35 \mu \mathrm{m}$ cell strainer. Dissociated single cells were then stained with $\mathrm{AO} / \mathrm{PI}$ for viability assessment using Countstar Fluorescence Cell Analyzer.

\section{Single-Cell Sequencing}

The scRNA-seq libraries were generated using the 10X Genomics Chromium Controller Instrument and Chromium Single Cell 3' V3 Reagent Kits (10X Genomics, Pleasanton, CA) according to the standard protocol provided in the manual. In brief, cells were adjusted to 1000 cells/uL and loaded into each channel to generate single-cell Gel Bead-In-Emulsions (GEMs). After the RT step, GEMs were broken and barcoded CDNA was purified and amplified. The amplified barcoded cDNA was fragmented, poly A-tailed, ligated with adaptors and index PCR amplified. The final libraries were quantified using the Qubit High Sensitivity DNA assay (Thermo Fisher Scientific) and the size distribution of the libraries were determined using a High 
Sensitivity DNA chip on a Bioanalyzer 2200 (Agilent). All libraries were sequenced by Illumina Sequencer (Illumina, San Diego, CA) on a 150 bp paired-end run.

\section{Single-Cell Sequencing Data Processing}

scRNA-seq data analysis was performed by NovelBio Bio-Pharm Technology Co., Ltd. with NovelBrain Cloud Analysis Platform. Cellranger software (version 3.0.0) was used to generate fastp (10) files with default parameters and to align reads to the mouse genome (mm10 Ensemble: version 92). Cells containing over 200 expressed genes with mitochondria UMI rate below 20\% were kept and mitochondria genes were removed. We applied the doubletCell function in the scran package to mark doublet cells and set the percentage of doublet cells at $5 \%$.

Seurat package (version 2.3.4, https://satijalab.org/seurat/) was used for data normalization and analysis. Principal Component Analysis (PCA) was generated based on the scaled data with top 2000 highly variable genes. The first 10 principal components were selected as input for t-distributed stochastic neighbor embedding (tSNE) construction and UMAP construction. We used the function "FindAllMarkers" in Seurat to identify differentially expressed genes in each cell cluster with Wilcox rank sum test algorithm under the following criteria: 1 . $\operatorname{lnFC}>0.25 ; 2$. $\mathrm{P}$ value $<0.05 ; 3$. min.pct $>0.1$. For further exploration, cells of the same cell type were selected for retSNE analysis, graph-based clustering and marker analysis.

\section{Gene Ontology Analysis}

We downloaded the GO annotations from NCBI (http://www. ncbi.nlm.nih.gov/), UniProt (http://www.uniprot.org/) and Gene Ontology (http://www.geneontology.org/) (11). Fisher's exact test was applied to identify significant GO categories and FDR was used to correct the $\mathrm{P}$ values.

\section{Pathway Analysis}

We performed pathway analysis according to KEGG database. We used Fisher's exact test to select significant pathways, and the threshold of significance was defined by P value and FDR (12).

\section{Quantitative Set Analysis for Gene Expression Analysis}

QuSAGE analysis was performed as described (13).

\section{Gene Co-Regulation Analysis}

We used the "find_gene_modules" function of Monocle 3 with default parameters to identity gene co-regulation network (14).

\section{Immunohistochemistry}

After deparaffinization with dimethylbenzene, tissue sections were sequentially rehydrated with graded alcohols. The antigens were retrieved by boiling the sections in citratedisodium hydrogen phosphate buffer $(\mathrm{pH}$ 6.0) with high pressure for 5 minutes. Endogenous peroxidase was inactivated by incubation with $0.3 \%$ hydrogen peroxide for 30 minutes. The slides were subsequently incubated with the primary antibody against FAT10 (ab134077, Abcam, USA) overnight at $4^{\circ} \mathrm{C}$, incubated with the biotinylated secondary antibody and streptavidin peroxidase (Invitrogen, Grand Island, USA) for 30 minutes at $37^{\circ} \mathrm{C}$, and developed with $3,3^{\prime}$-diaminobenzidine solution (GeneTech, Shanghai, China) before counter-staining with hematoxylin.

\section{Statistical Analysis}

Statistical analysis was performed using SPSS v19.0 software (Chicago, IL, USA). Student t test or Wilcoxon rank-sum test was used. A value was considered statistically significant if $\mathrm{P}<0.05$.

\section{RESULTS}

\section{scRNA-seq Identifies 21 Distinct Cell Types in Mouse Heart Allograft}

To comprehensively analyze cell populations in acute rejection at the single-cell level, we performed 4 cases of heterotopic mouse heart transplantation (2 syngeneic and 2 allogeneic) and collected grafts on Day 5. For each graft, single-cell RNA profiling was performed using the 10x Chromium platform. In an initial quality control, on average, we detected transcripts from 2,033 different genes with 51,882 sequencing reads per cell in the merged data set. In total, we acquired 18,698 cells from 2 allogeneic heart grafts and 19,904 cells from 2 syngeneic heart grafts (Supplementary Figure 1). With unsupervised clustering analysis using Seurat based on shared and unique patterns of gene expression, we identified 21 distinct cell clusters (Figure 1A). As expected, syngeneic grafts and allogeneic grafts displayed different patterns of cell populations (Figure 1B). We annotated cell clusters in accordance with signature gene expression and literature. In essence, we identified cardiomyocytes $\left(1.80 \%, \mathrm{Tnt}^{+}, \mathrm{Fabp}^{+}\right)$, five types of endothelial cells $\left(46.52 \%, C d h 5^{+}, G p i h b p 1^{+}\right)$, three fibroblast populations $\left(26.29 \%, \mathrm{Dcn}^{+}, \mathrm{Bgn}^{+}\right)$, T/NK cells $\left(1.36 \%, \mathrm{Cd} 3 d^{+}\right.$, $\left.\mathrm{Cd} 3 g^{+}, \mathrm{Nkg}^{+}, \mathrm{Gzma}^{+}\right)$, five types of macrophages $(14.01 \%$, $\left.\mathrm{Cl}_{\mathrm{qc}} \mathrm{c}^{+}, \mathrm{Ctsc}^{+}\right)$, two granulocyte populations $\left(3.27 \%, \mathrm{~S} 100 \mathrm{a} 9^{+}\right.$, $\left.S 100 a 8^{+}\right)$, B cells $\left(1.24 \%, L y 6 d^{+}, C d 79 a^{+}\right)$, smooth muscle cells $\left(2.09 \%, M y l k^{+}\right)$, pericytes $\left(3.37 \%, K_{c n j} 8^{+}\right)$and an undefined cell population (0.05\%, unknown) (Figures 1A, C). The full set of raw data of this study has been deposited in NCBI's Gene Expression Omnibus (GEO) and is available through the GEO accession number GSE151048.

\section{Activation of T Cells and NK Cells in Allograft Rejection}

It is known that $\mathrm{T}$ cells and NK cells play a significant role in acute rejection. We made further analysis of T/NK cells with single-cell transcriptomes. Re-clustering of T/NK cells revealed seven $\mathrm{T}$ cell populations and two clusters of NK cells (Figure 2A). Cluste1,2,4,7 were characterized as CD8+ T cells due to the expression of CD8a. We also observed that Cluster 1 expressed genes previously associated with central memory $\mathrm{T}$ cells (Lef1, Cd69, Tcf7), and Cluster 2 expressed genes associated with effector T cells (Zeb2, Tnf, Ifng) (Supplementary Figure 2) (15). Combining the expression of signature marker genes, cellproliferating gene (Mki67) and differentially expressed genes, we 


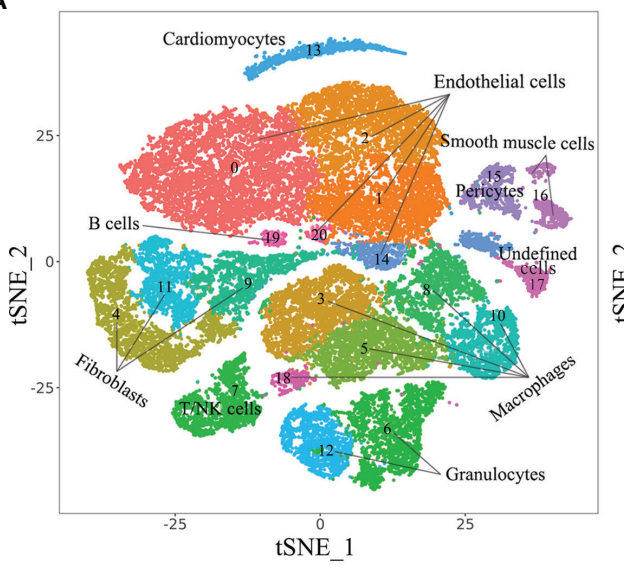

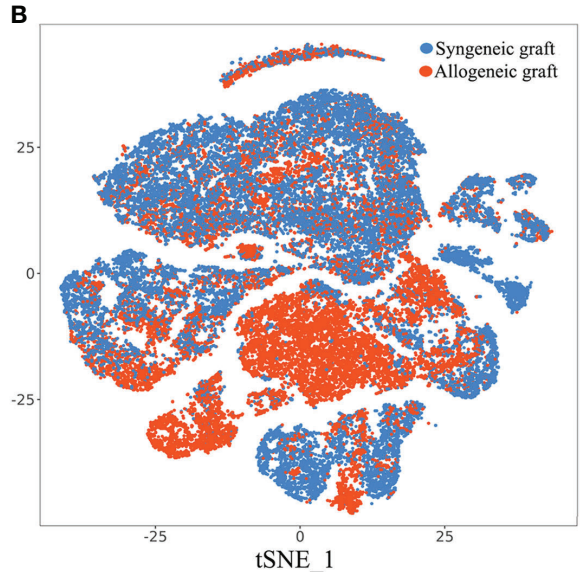

C

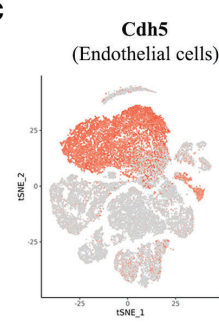

Cd3g
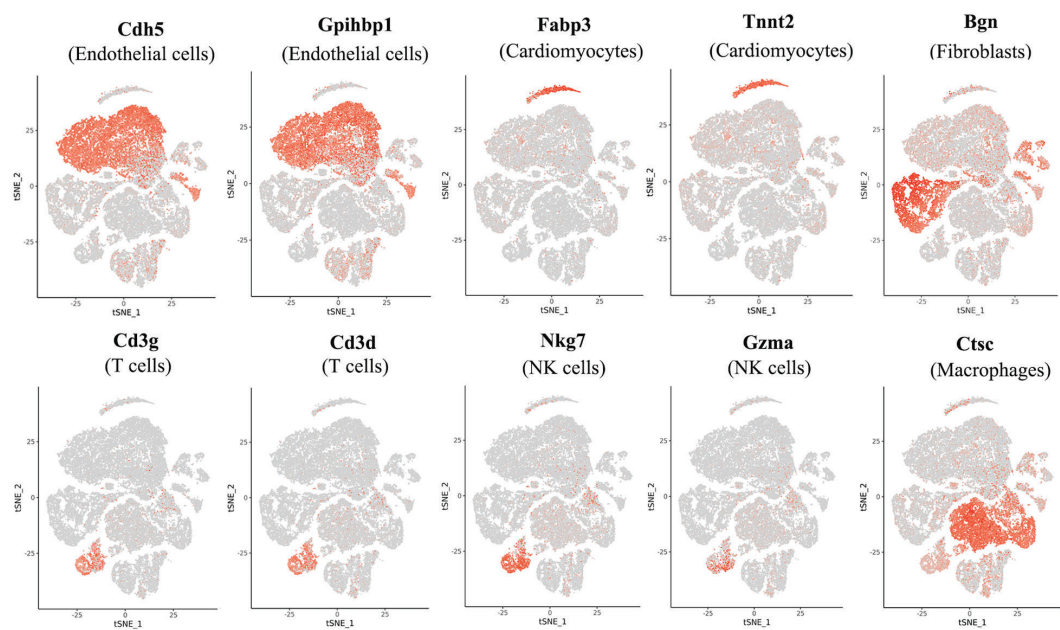

Gzma

(NK cells)
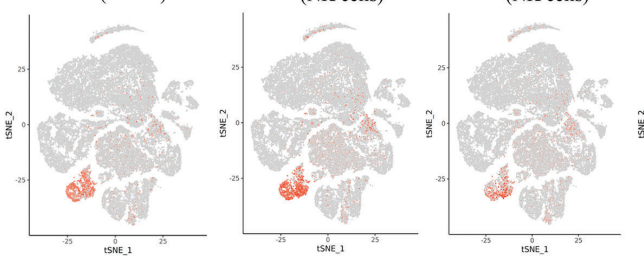

Ctsc

(Macrophages)

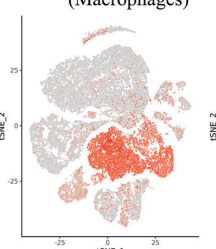

Mylk
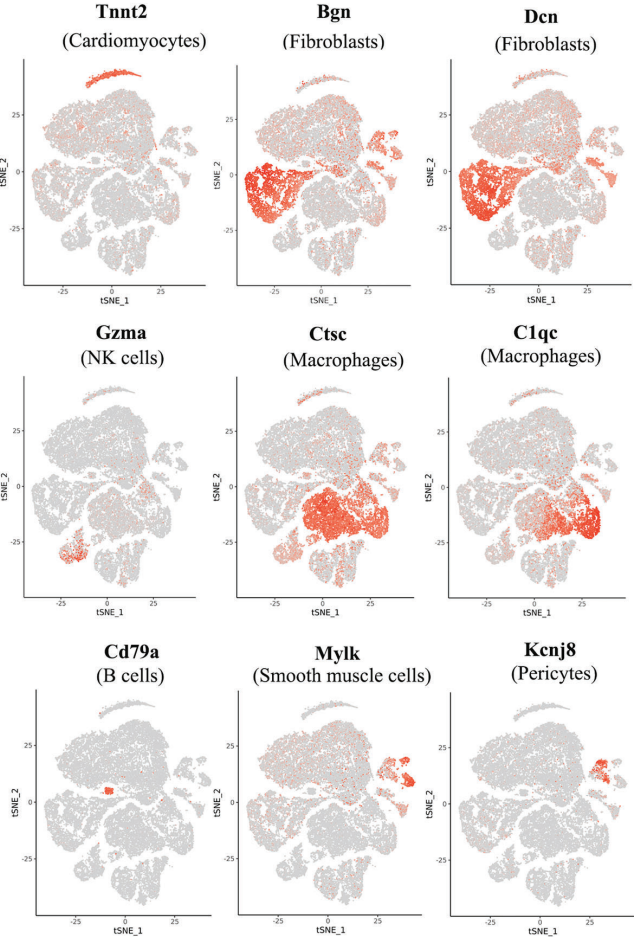

C1qc
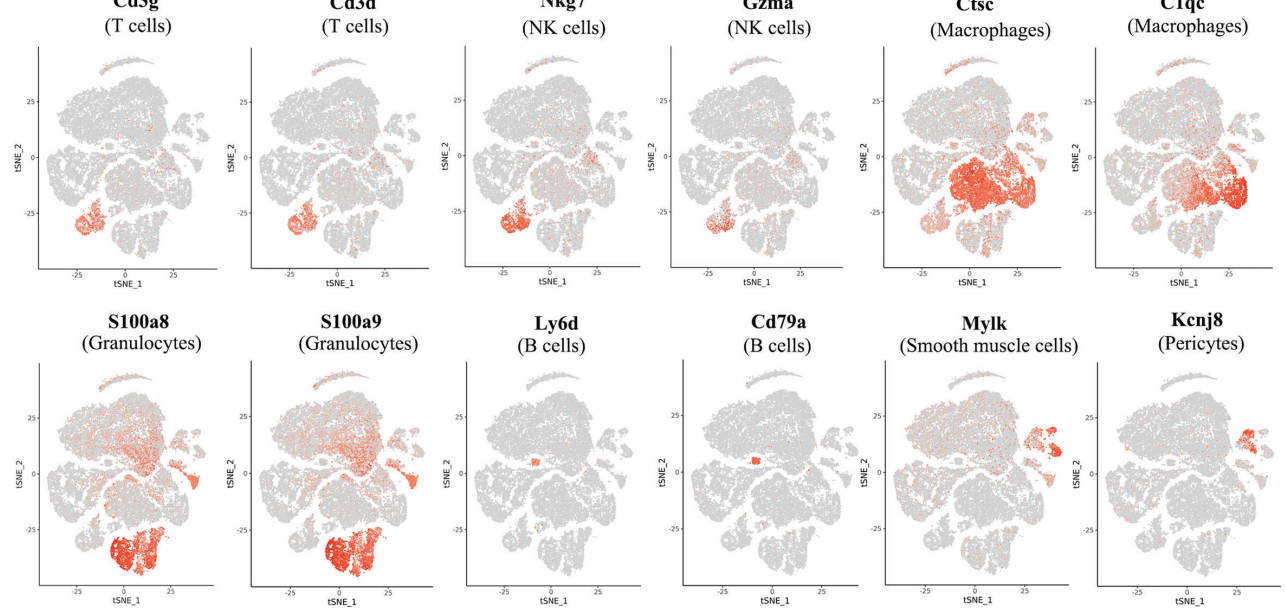

FIGURE 1 | Comprehensive scRNA-seq analysis of cell types in syngeneic and allogeneic heart grafts. (A) Single-cell transcriptomes of cardiac cells in two syngeneic and two allogeneic heart grafts collected on Day 5 post-transplant were analyzed with an unsupervised dimensionality reduction algorithm (Seurat) to identify groups of cells with similar gene expression pattern. Each dot stands for a cell. (B) Distribution of cells originating from syngeneic graft or allogeneic graft as indicated in (A). (C) Canonical cell markers were used to characterize cell clusters in the t-SNE plot, including endothelial cells, cardiomyocytes, fibroblasts, T cells, macrophages, granulocytes, B cells, smooth muscle cells and pericytes.

annotated these cell clusters as Central memory $\mathrm{CD}^{+} \mathrm{T}$ cells, Effector $\mathrm{CD} 8^{+} \mathrm{T}$ cells, Activated $\mathrm{CD} 4^{+} \mathrm{T}$ cells, Activated $\mathrm{CD} 8^{+} \mathrm{T}$ cells, Activated NK cells, Resting $\mathrm{CD} 4^{+} \mathrm{T}$ cells, Resting $\mathrm{CD} 8^{+} \mathrm{T}$ cells, Resting NK cells and Treg cells (Figures 2A-C). Additionally, a large number of metabolic pathways were stimulated in Activated $\mathrm{CD} 4^{+} \mathrm{T}$ cells, $\mathrm{CD} 8^{+} \mathrm{T}$ cells and $\mathrm{NK}$ cells, such as glycolysis/gluconeogenesis pathway, oxidative phosphorylation pathway, pyrimidine metabolism pathway and purine metabolism pathway (Figure 2D), which are involved in graft rejection (16). Unsurprisingly, Activated T cells and NK cells predominantly comprised cells from allogeneic grafts, whereas Resting $\mathrm{T}$ cells predominantly comprised cells from syngeneic grafts (Figure 2E). Furthermore, we found enhanced expression of granzyme B (Gzmb) and interferon- $\gamma$ (Ifng) in allogeneic heart grafts (Figure 2F), which is consistent with previous reports $(17,18)$. 
A

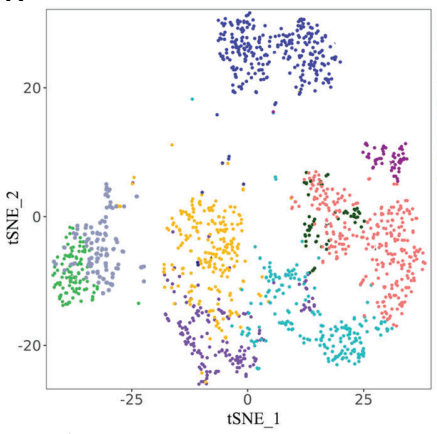

C

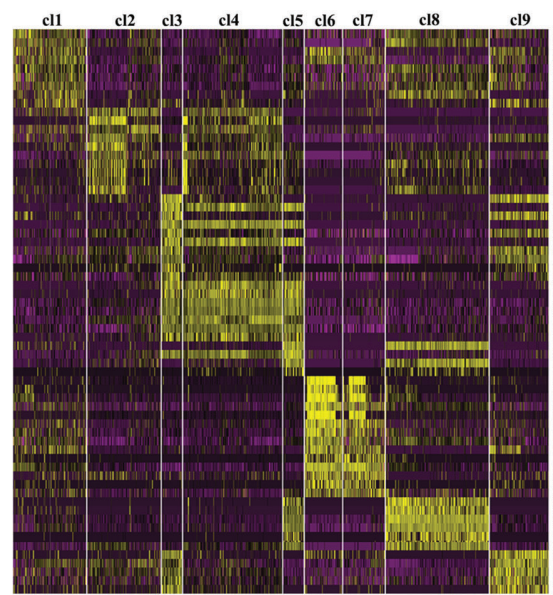

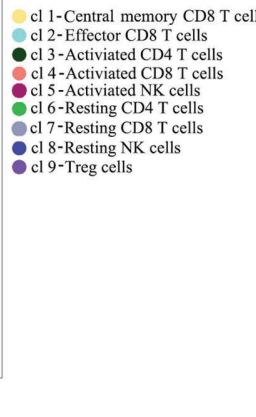
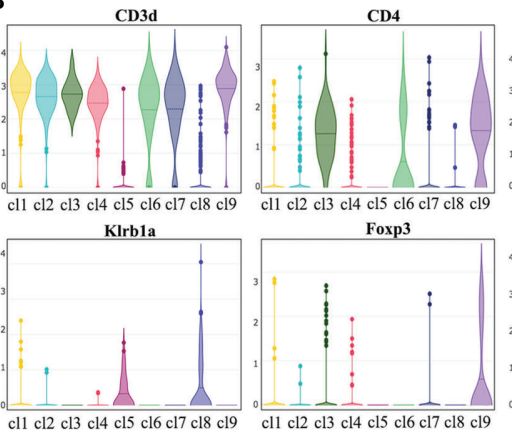

Foxp3

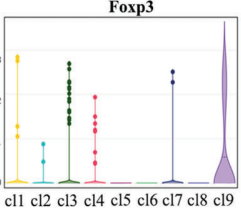

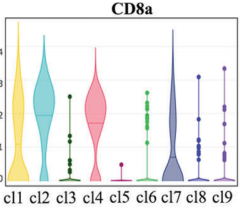

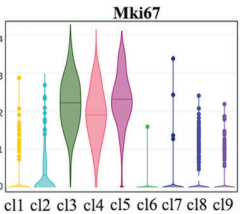

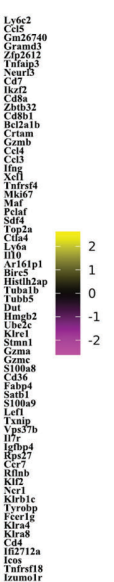

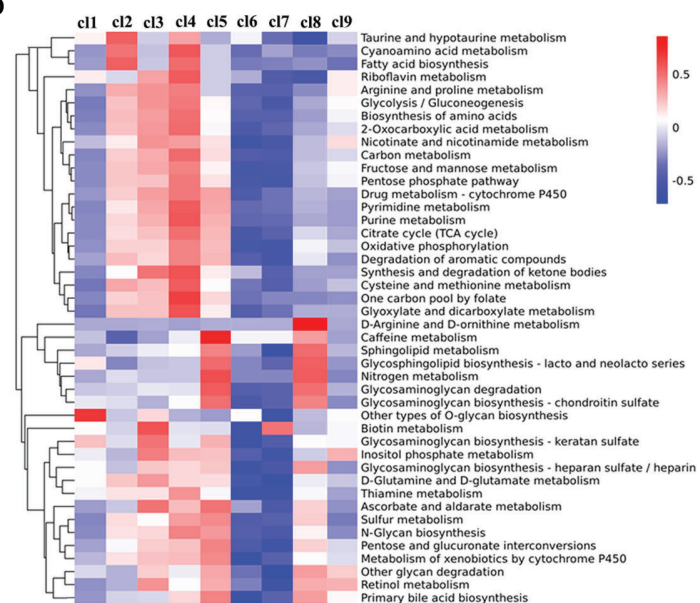

$\mathbf{F}$
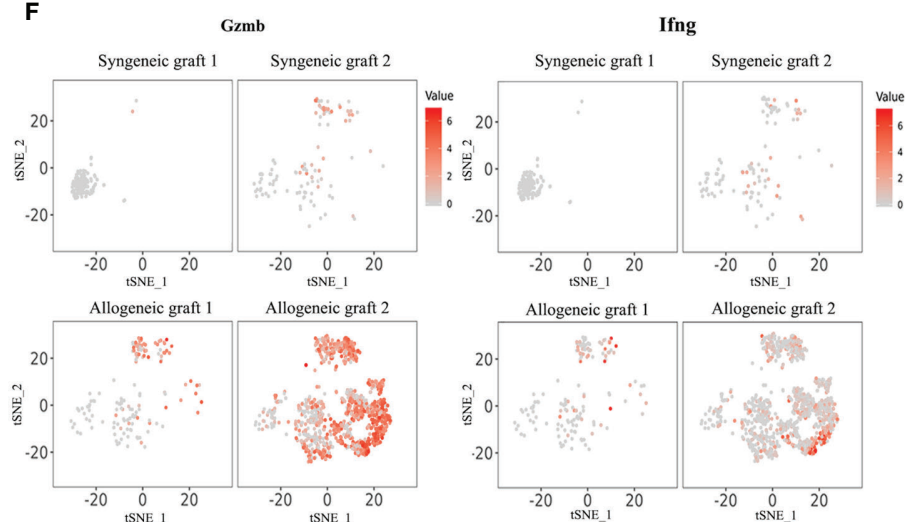

FIGURE 2 | Characterization of T cells and NK cells. (A) Reclustering of T/NK cells with the t-SNE plot identifying seven T cell populations and two NK cell populations. (B) Violin plots displaying the expression of representative cell markers (CD3d for T cells, CD4 for CD4 ${ }^{+} \mathrm{T}$ cells, CD8a for CD8 ${ }^{+} \mathrm{T}$ cells, Klrb1a for NK cells, Foxp3 for Treg cells, Mki67 for proliferating cells). The y axis shows normalized read counts. (C) Heatmap of all T/NK cells clustered by recursive hierarchical clustering and Louvain-Jaccard clustering (Seurat) showing gene expression signatures in each cell population. (D) Quantitative Set Analysis for Gene Expression (QUSAGE) analysis examining active cellular metabolic pathways in each T/NK cell population. (E) Proportion of cells in each T/NK cell cluster from allogeneic or syngeneic graft. (F) Expression of Gzmb and Ifng in the allogeneic and syngeneic heart grafts in the t-SNE plots as shown in (A).

\section{Macrophage Heterogeneity in Allograft Rejection}

Currently, most immunosuppressive drugs target $\mathrm{T}$ cells. However, more and more studies demonstrate that innate immune cells play a more complex role (1). Monocyte infiltration is quantitatively associated with kidney allograft dysfunction during acute rejection (19). In our analysis, macrophages represent the largest immune cell population in allogeneic grafts, constituting 70.5\% of total immune cells.

Re-clustering of macrophages revealed five subsets $(\mathrm{m} 1 \sim \mathrm{m} 5)$ (Figure 3A). Among them, $\mathrm{m} 1$ and $\mathrm{m} 2$ were characterized as resident macrophages due to the expression of $C \times 3 c r 1$ and $F 13 a 1$ 
A

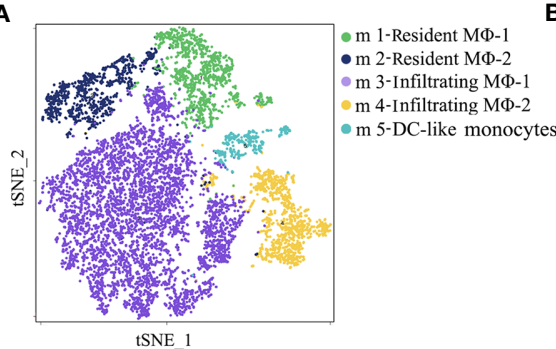

D

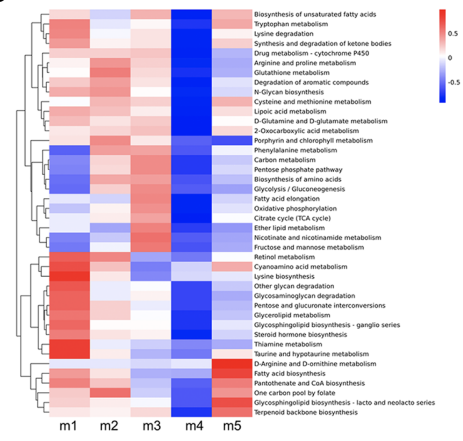

G
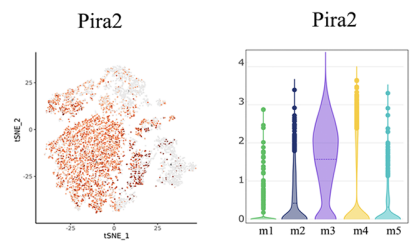

H

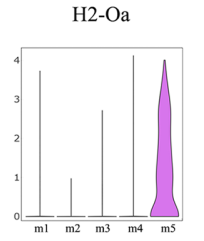

$\mathrm{H} 2-\mathrm{DMb} 2$

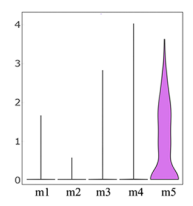

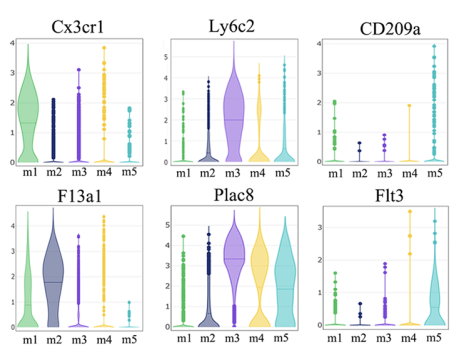

E
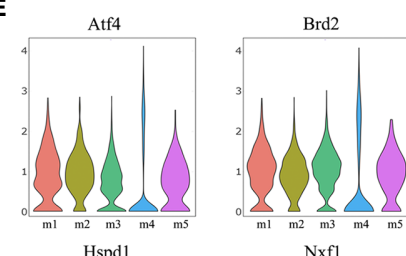

Nxfl
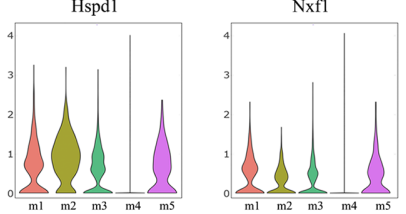

c
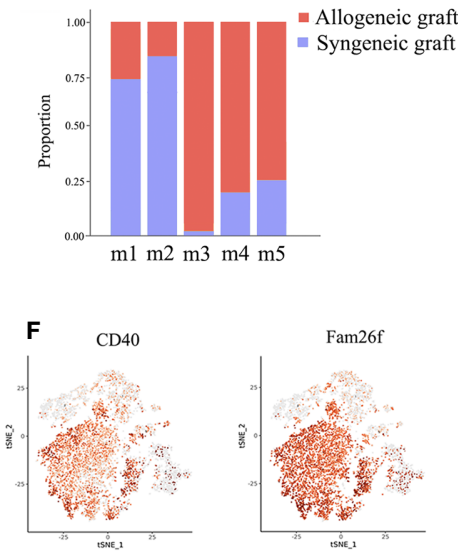

CD40
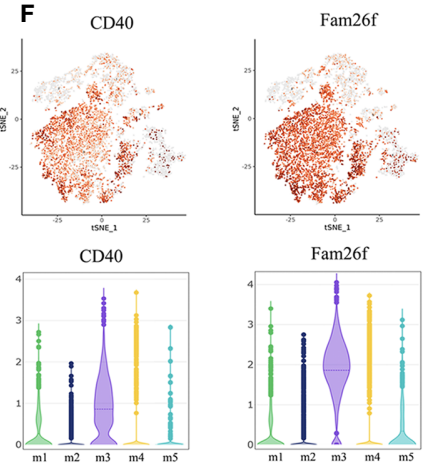

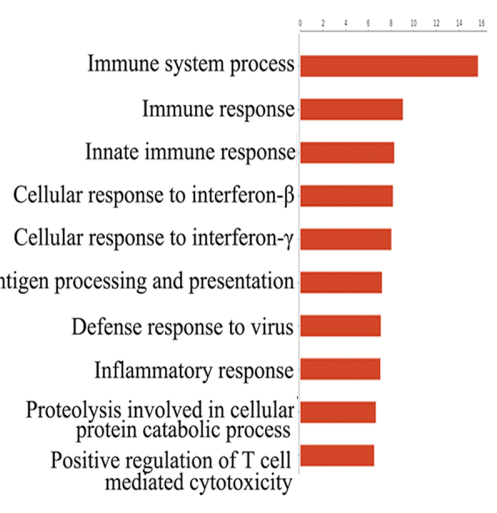

J

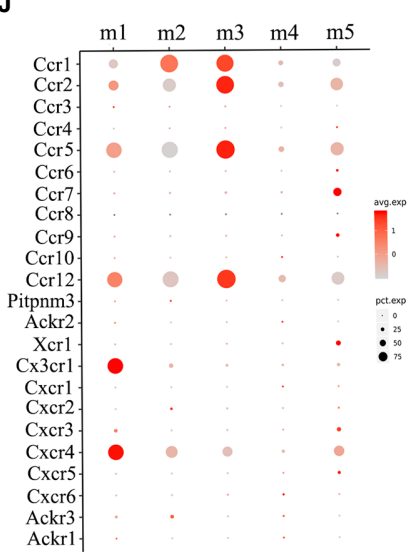

FIGURE 3 | Annotation of macrophage subsets. (A) t-SNE plot identifying five distinct populations of macrophages from two allogeneic heart grafts and two syngeneic heart grafts. (B) Violin plots displaying the expression of representative markers across macrophage types (Cx3cr1 and F13a1 for resident macrophages, Ly6c2 and Plac8 for infiltrating macrophages, CD209a and Flt3 for dendritic cells). (C) Proportion of cells in each macrophage population from allogeneic or syngeneic graft. (D) Quantitative Set Analysis for Gene Expression (QUSAGE) analysis examining active cellular metabolic pathways in each macrophage population. (E) Violin plots displaying the expression of genes (Atf4, Brd2 Hspd1, Nxf1) associated with cell proliferation, metabolism and activation in each macrophage population. (F) t-SNE maps and violin plots indicating the expression of CD40 and Fam26f. (G) t-SNE maps and violin plots indicating the expression of Pira2. (H) Violin plots displaying the expression of MHC class II molecules H2-Oa and H2-DMb2. (I) GO Biological Process and Pathway Analysis of the m3 cluster. (J) A dot plot showing the expression of the chemokine receptor in macrophages subsets.

$(20,21)$, while $\mathrm{m} 3$ and $\mathrm{m} 4$ were considered as infiltrating macrophages as indicated by the expression of $L y 6 c 2$ and Plac8 (22). CD209a is a marker for monocyte-derived dendritic cells (DC) (23) and Flt3 is a key regulator for the development of dendritic cells (Figure 3B) (24). Therefore, we annotated $\mathrm{m} 5$ as DC-like monocytes. Consistent with previous reports that infiltrating macrophages are associated with rejection, $\mathrm{m} 3$ and $\mathrm{m} 4$ clusters were predominantly from allogeneic grafts, while $\mathrm{m} 1$ and $\mathrm{m} 2$ clusters were majorly from syngeneic allografts (Figure 3C). Furthermore, on one hand, $\mathrm{m} 1$ and $\mathrm{m} 2$ adopted
M2 macrophage phenotype as shown by a panel of M2 markers, including Mrc1, Folr2 and Cbr2, suggesting a potential pro-repair role. On the other hand, $\mathrm{m} 3$ and $\mathrm{m} 4$ belonged to $\mathrm{M} 1$ macrophages as indicated by the expression of M1 proinflammatory chemokines and cytokines, such as Cxcl10 and $\operatorname{Tnf}$ (Supplementary Figure 3) (25).

Surprisingly, analysis of cellular metabolic pathways demonstrated that different from the other four clusters, the m4 cluster was metabolically "silent" (Figure 3D), which was further confirmed by the minimal gene expression of factors 
related to cell proliferation, metabolism and activation, such as Atf4, Brd2, Hspd1 and Nxf1 (Figure 3E) (26), suggesting that m3, not $\mathrm{m} 4$, was the subset of infiltrating macrophages actively associated with graft rejection. It is possible that the $\mathrm{m} 4$ cluster may be in a transition state, waiting to be activated. In support of this interpretation, the costimulatory receptor CD40 and another membrane molecule Fam26f were expressed at a much higher level in $\mathrm{m} 3$ than in $\mathrm{m} 4$ (Figure 3F). A recent study reported that murine macrophages acquire alloantigen-specific memory, which requires the interaction between MHC-I and PIR-A (27). Interestingly, Pira2 was highly expressed in $\mathrm{m} 3$ (Figure 3G). Moreover, the $\mathrm{m} 3$ cluster was also characterized by enriched expression of rejection-associated genes, such as Cxcl9, Gzmb, Psmb9, Isg20, Tap1 and Nampt (Supplementary Figure 4) (28-30). We further performed GO enrichment analysis with the transcriptome of $\mathrm{m} 3$ and found enrichment of genes involved in immune response activation, such as immune response, inflammatory response and positive regulation of $\mathrm{T}$ cell mediated cytotoxicity (Figure $3 \mathrm{I}$ ). We further analyze the chemokine receptor expression in macrophages, interestingly, Ccr1, Ccr2, Ccr5 and Ccr12 was highly expressed in $\mathrm{m} 3$ (Figure 3J), and these C-C motif chemokine receptors may contribute to $\mathrm{m} 3$ infiltration. Taken together, the m3 cluster was the cell population linked to acute rejection.

Dendritic cells are important antigen presenting cells. From this perspective, we further found that the m5 cluster highly expresses some dendritic cell markers, such as Cd209a and Flt3 (Figure 3B), it also highly expressed MHC-II molecules, such as $\mathrm{H} 2-\mathrm{Oa}$ and $\mathrm{H} 2-\mathrm{DMb} 2$ (Figure $\mathbf{3 H}$ ). As shown in Figure 3C, the $\mathrm{m} 5$ was predominantly composed of cells from allogeneic grafts, it therefore may presents the characteristics of antigen presenting cells.

\section{Characterization of Endothelial Cells and Identification of a Rejection-Related Cluster}

Endothelial cells represented the largest cell population in our analysis, constituting $46.52 \%$ of total cells. Re-clustering of all the endothelial cells from both syngeneic and allogeneic grafts unveiled five types (EC1 EC5) (Figure 4A). The largest population, EC1, was characterized as capillary endothelial cells due to the expression of Cd300lg. EC2 expressed the canonical arterial endothelial cell marker Stmn2, while EC3 expressed the venous endothelial cell marker Nr2f2. EC4 highly expressed a smooth muscle actin gene Acta2, suggesting they were fibroblast-like endothelial cells. The EC5 population expressed Vcam1, and likely represented microvascular endothelial cells (Figure 4B) $(31,32)$. Of note, the EC5 cluster was almost exclusively composed of cells from allogeneic grafts (Figure 4C). Consistently, GO enrichment analysis and KEGG pathway analysis of EC5 indicated activation of pathways closely related to graft rejection, including antigen processing and presentation, immune system process and allograft rejection (Figures 4D, E). More strikingly, a panel of MHC-II molecules (H2-Aa, H2-DMb1, H2-Eb1, H2-DMa, H2-Ab1, etc.) were predominantly expressed in the EC5 cluster (Figure 4F), suggesting that EC5 may exert its function in presenting donor alloantigens to host $\mathrm{T}$ cells to mediate transplant rejection.

\section{Ubd Is Specifically Upregulated in Endothelial Cells During Graft Rejection}

Since the cluster EC5 was almost exclusively present in allogeneic graft, we made further analysis of EC5 to identify rejectionspecific markers. Excitingly, compared to other cell types or other endothelial cell clusters, only almost all the cells in EC5 highly expressed Ubiquitin D (Ubd) gene (Figures 5A, B) (33). We next analyzed co-expression genes with $U b d$ and revealed that many of them, such as Cxcl9, Cxcl16, Ly6a, Batf2, Batf3 and H2-Q2, were genes which play an important role in regulating immune cells, including $\mathrm{T}$ cells, macrophages and DCs (Figure 5C). In support of this scenario, pathway analysis indicated the enrichment of genes involved in pathways associated with immune response, such as immune system process, antigen processing and presentation of exogenous peptide antigen via MHC class II, and immune response (Figure 5D), suggesting Ubd positive endothelial cells activate immune system to mediate graft rejection. The specific upregulation of Ubd in graft rejection was confirmed by immunohistochemistry staining. Ubd was significantly upregulated in endothelial cells in allogeneic heart grafts compared to syngeneic heart grafts (Figure 6A).

To investigate clinical relevance, we collected 10 human kidney biopsy specimens (5 with rejection, 5 without rejection, Supplementary Table 1). Immunohistochemistry staining showed that massive endothelial cells were Ubd positive in the kidney grafts with rejection. In contrast, staining was sparse or absent in kidney grafts with no injury (Figure 6B). These results indicate that Ubd positive cells are closely associated with acute rejection, and Ubd could be considered as a biomarker to predict transplant rejection in the clinic.

\section{DISCUSSION}

This study provides a comprehensive single-cell atlas of gene expression involved in acute rejection using the classical mouse heterotopic heart transplant model. Consistent with previous studies, we confirmed activation and expansion of T cells and NK cells. Moreover, among the five subsets of macrophages we identified, infiltrating macrophages ( $\mathrm{m} 3$ and $\mathrm{m} 4$ ), rather than resident macrophages ( $\mathrm{m} 1$ and $\mathrm{m} 2$ ), were associated with graft rejection. Interestingly, metabolic pathway analysis showed that the $\mathrm{m} 4$ cluster was in an inactive state. Our results showed $\mathrm{m} 3$ and $\mathrm{m} 4$ cluster expressed pro-inflammatory chemokines and cytokines Cxcl10 and Tnf, two potent M1 macrophage activation markers. Moreover, the expression of activating molecules, such as CD40 and Fam26f, was elevated in $\mathrm{m} 3$, but not in $\mathrm{m} 4$. CD40 is a costimulatory molecule, which plays an important role in immune cell activation and survival. Fam26f is a conserved surface molecule, which also modulates immune responses. Specifically, it has been reported that FAM26F is an activation 
A

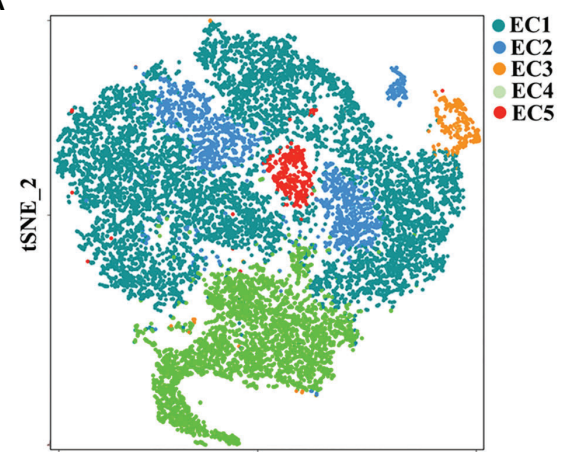

tSNE_1

C

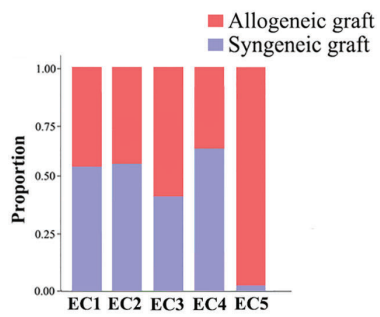

D

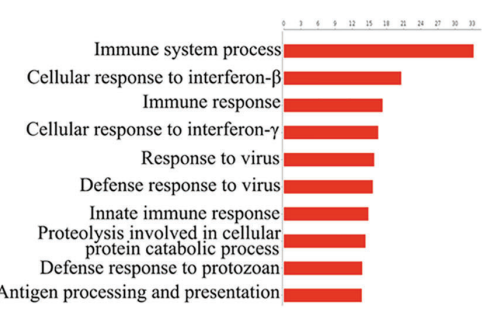

B

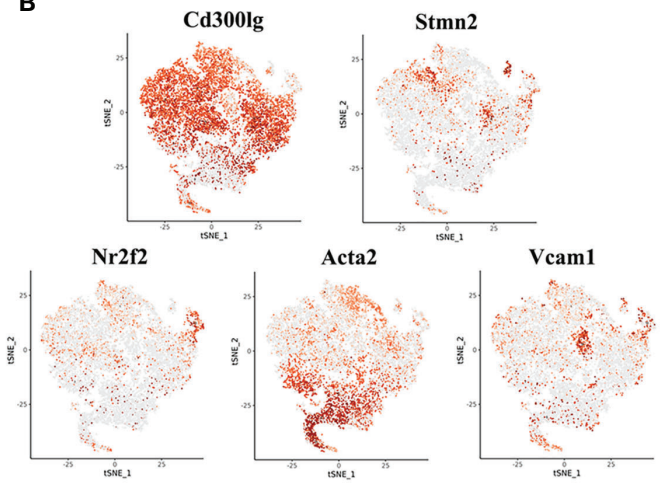

E

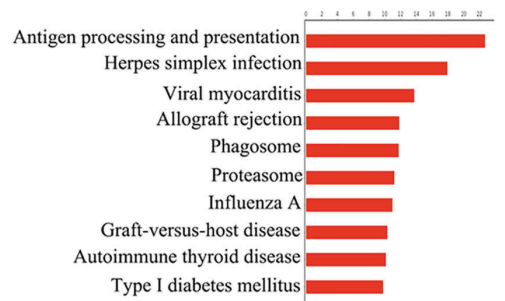

F

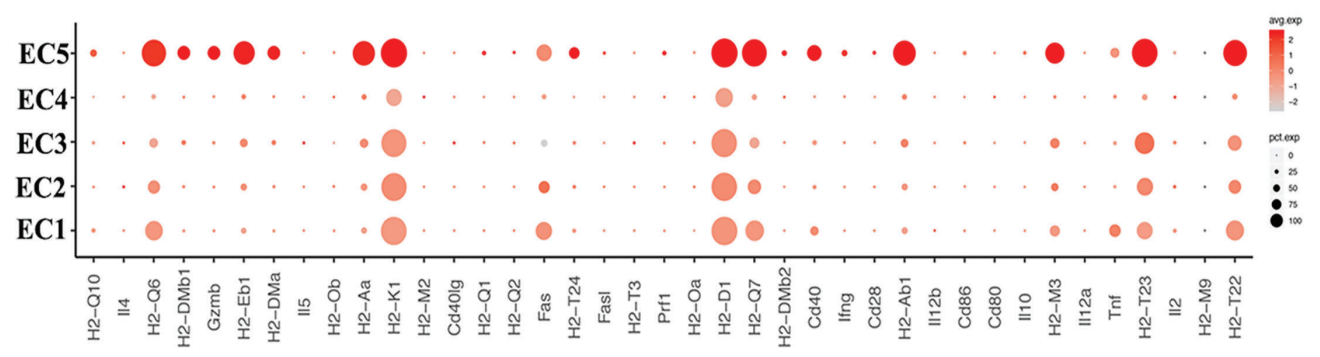

FIGURE 4 | Analysis of endothelial cell (EC) subsets. (A) Reclustering of endothelial cells identifying five subsets. (B) Expression of canonical cell markers in each EC cluster (EC1-EC5) in the t-SNE plots. (C) Proportion of cells in each EC subset from allogeneic or syngeneic graft. (D) GO enrichment analysis of EC5. (E) KEGG pathway analysis of EC5. (F) A dot plot showing the expression of inflammatory genes and MHC class II molecules in each EC subset.

marker between resting macrophages and lipopolysaccharide (LPS) activated macrophages, and that the up-regulation of FAM26F is related to early liver graft failure (34). Therefore, further studies are required to distinguish the functional and mechanistic difference between $\mathrm{m} 3$ and $\mathrm{m} 4$, which may shed light on how macrophages are recruited to the allograft and activated. A recent study demonstrated that mouse monocytes and macrophages may acquire alloantigen-specific memory in a PIR-A dependent way. Intriguingly, our analysis also identified the up-regulation of Pira2 in the $\mathrm{m} 3$ cluster, suggesting that the m3 cluster may also function in "trained immunity" as memory cells.

In addition to macrophage surface markers, the $\mathrm{m} 5$ cluster also highly expressed MHC-II molecules and presented the characteristics of antigen presenting cells. Previous investigations have shown that monocyte-derived DCs isolated from the allograft function as potent antigen-presenting cells that could drive both $\mathrm{T}$ cell proliferation and interferon $\gamma(\mathrm{IFN}-\gamma)$ production, while depletion of monocyte-derived DCs significantly alleviates rejection $(35,36)$. Thus, the $\mathrm{m} 5$ cluster was also an important group of macrophages involved in rejection. Taken together, the characterization of these macrophage populations provides a valuable framework for studying the role of macrophages in graft rejection and related mechanisms.

Recent studies using single-cell transcriptome profiling have revealed the existence of extensive heterogeneity in ECs in many settings, including tumors, acute lung injury, as well as normal organs, which contribute to the understanding of EC diversity (31, 37, 38). scRNA-seq analysis of a human kidney biopsy specimen identified three endothelial cell groups and one of them was associated with antibody-mediated rejection (8). Our analysis identified 5 populations of ECs (EC1 EC5) in acute rejection. Excitingly, a novel microvascular endothelial cell population (EC5) almost uniquely stemmed from allogeneic heart grafts. In the context of organ transplantation, 
A

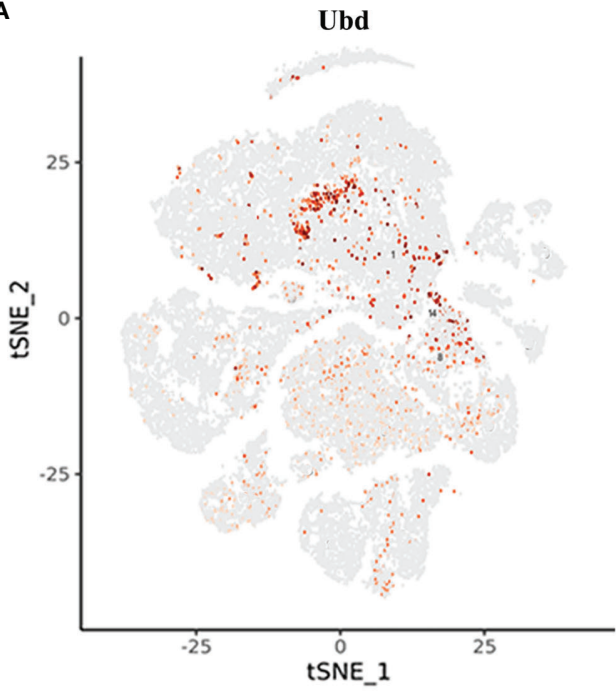

C

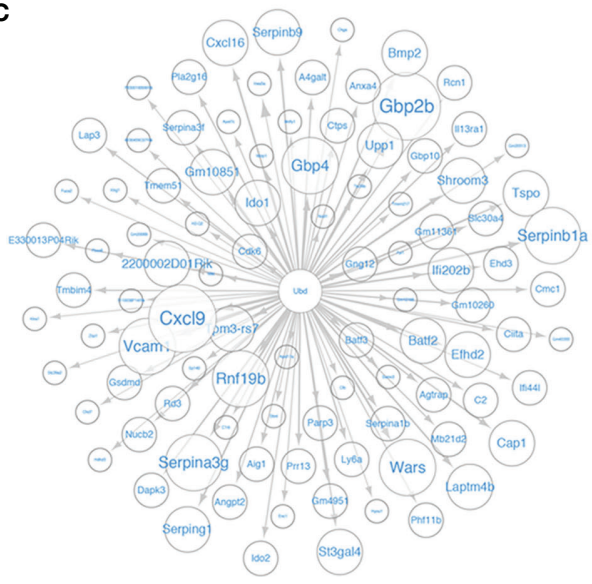

B

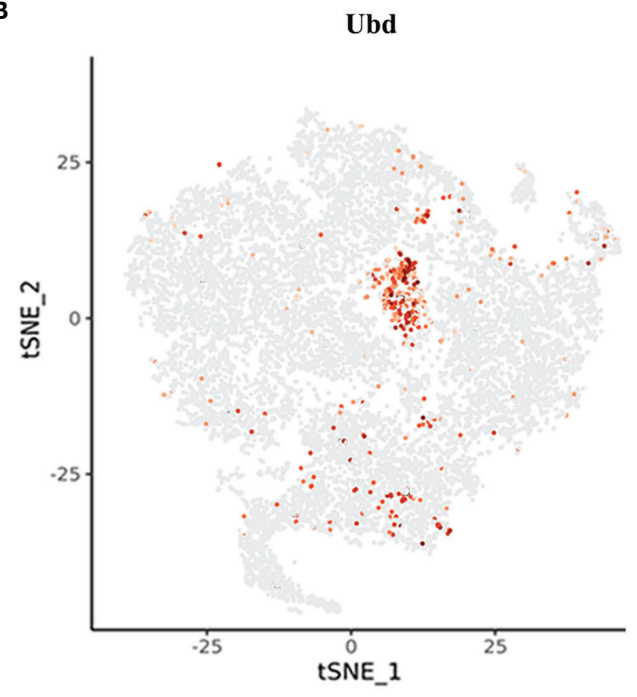

D

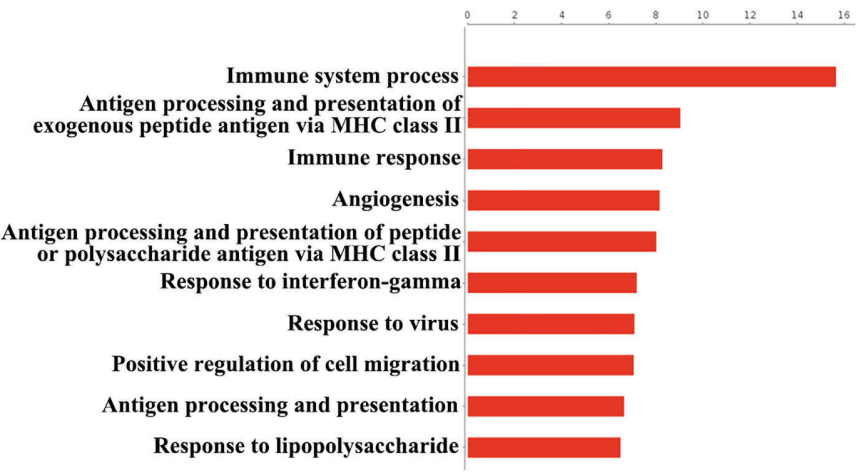

FIGURE 5 | Identification of Ubd in EC5. (A) t-SNE map (from Figure 1A) indicating the expression of Ubd in all the cells. (B) t-SNE plot indicating the expression of Ubd in the re-clustered endothelial cells. (C) Correlation network analysis of Ubd displaying its co-expression genes in endothelial cells. (D) GO enrichment analysis with Ubd-positive ECs and Ubd-negative ECs.

endothelial cells express both MHC class I and class II molecules, enabling them to present antigens to recipient $\mathrm{T}$ cells. Consistent with that, ablation of MHC molecules on ECs mitigates T cellmediated rejection $(39,40)$. Interestingly, the allograft-specific EC5 cluster exhibited significant up-regulation of genes involved in processes related to antigen processing and presentation, graft rejection and immune system process. Furthermore, we observed that MHC-II molecules were predominantly expressed in the EC5 cell cluster, suggesting that EC5 is the endothelial cell population that exerts the antigen-presenting capacity. Thus, our investigation broadens the horizon of the role of endothelial cells in transplantation by pinpointing EC5 as the endothelial cell group responsible for rejection.

Since EC5 was uniquely present in allogeneic heart grafts, we performed more analysis to characterize EC5 in order to identify new diagnostic and therapeutic targets. We found that $U b d$ was specifically up-regulated in EC5. Ubd (also called Fat10) is a ubiquitin-like protein that is capable of inducing ubiquitinindependent degradation of proteins via the proteasome (33). As for immune responses, Ubd was initially found to be expressed in mature B lymphocytes and dendritic cells, which is involved in antigen presentation and regarded as a biomarker for immune activation (41). In the context of organ transplantation, bioinformatic meta-analysis of microarray datasets and bulk-RNA sequencing indicate that Ubd is related to rejection and has the potential to be considered as a diagnostic marker $(42,43)$, yet with no confirmation or further study. Our discovery provides a new perspective that Ubd is specifically upregulated in endothelial cells in acute rejection. Besides, we confirmed the up-regulation of Ubd in endothelial cells in acute rejection in mouse allogeneic hearts and human kidney biopsy specimens. In addition, gene correlation network characterization and functional enrichment analysis suggest that Ubd plays an important role in immune regulation. 
A

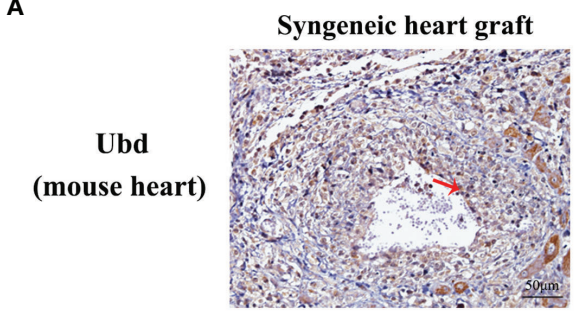

B

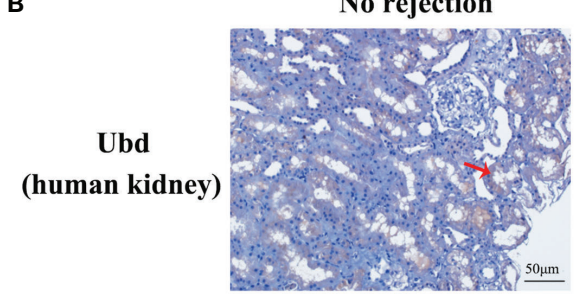

Allogeneic heart graft

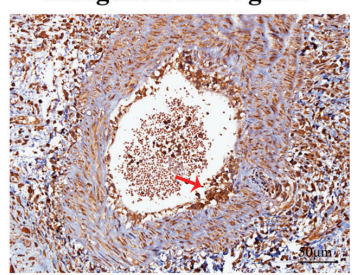

Rejection

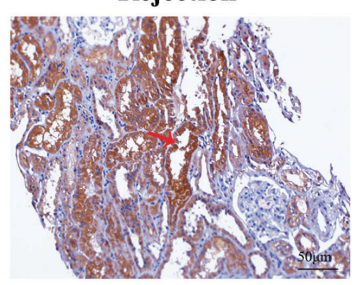

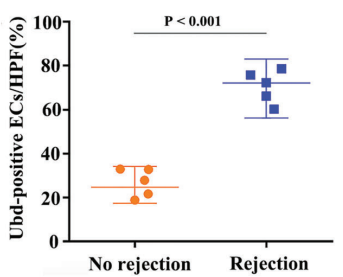

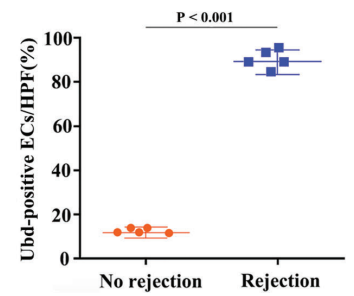

FIGURE 6 | Upregulation of Ubd in endothelial cells during rejection. (A) Representative immunohistochemistry staining of Ubd in the allogeneic and syngeneic mouse heart grafts. (B) Representative immunohistochemistry staining of Ubd in human kidney biopsy specimens with/without rejection. Arrows indicate positive staining in endothelial cells. Scale bars, $50 \mathrm{~mm}$. Quantitative analysis was shown on the right ( $\mathrm{n}=5$ for each group).

However, further investigation is needed to address the effect of Ubd expression and why Ubd is specifically induced in EC5, which may shed light on new therapeutic tools to prevent rejection.

In conclusion, we provide a comprehensive landscape of graft-infiltrating cells in acute rejection, as well as their intrapopulation heterogeneity. We reveal two graft-infiltrating macrophage populations and only one of them is actively involved in graft rejection. Meanwhile, we identify a novel endothelial cell cluster that may potentially exert antigenpresenting capacity to stimulate rejection. Furthermore, the expression of Ubd defines such endothelial cell cluster and could be used as a biomarker for acute rejection.

\section{DATA AVAILABILITY STATEMENT}

The datasets presented in this study can be found in online repositories. The names of the repository/repositories and accession number(s) can be found in the article/Supplementary Material.

\section{ETHICS STATEMENT}

The studies involving human participants were reviewed and approved by the Research Ethics Committee of the First Affiliated Hospital of Sun Yat-sen University. Written informed consent to participate in this study was provided by the participants' legal guardian/next of kin. The animal study was reviewed and approved by the Animal Care Ethics Committee of the First Affiliated Hospital of Sun Yat-sen University.

\section{AUTHOR CONTRIBUTIONS}

YT and XS designed the study and drafted the manuscript. YZ performed mouse heterotopic heart transplantation. YT and JW collected samples. MD carried out immunohistochemistry staining experiments. YT, YZ, JW, XH, CW and XS analyzed and interpreted data. JL, MC, YG and SL provided resources. All authors contributed to the article and approved the submitted version.

\section{FUNDING}

XS was supported by National Natural Science Foundation of China (31800753) and Guangdong Basic and Applied Basic Research Foundation (2019A1515010639). CW was supported by National Key Research and Development Program of China (2019YFA0111500). YG was supported by National Natural Science Foundation of China (31800758). The project was also funded by Guangdong Provincial Key Laboratory of Organ Donation and Transplant Immunology (2013A061401007, 2017B030314018, 2020B1212060026) and Guangdong Provincial International Cooperation Base of Science and Technology (Organ Transplantation) (2015B050501002, 2020A0505020003).

\section{SUPPLEMENTARY MATERIAL}

The Supplementary Material for this article can be found online at: https://www.frontiersin.org/articles/10.3389/fimmu.2022.832573/ full\#supplementary-material 


\section{REFERENCES}

1. Ochando J, Fayad ZA, Madsen JC, Netea MG, Mulder WJM. Trained Immunity in Organ Transplantation. Am J Transplant (2020) 20(1):10-8. doi: $10.1111 /$ ajt.15620

2. Li XC. The Significance of Non-T-Cell Pathways in Graft Rejection: Implications for Transplant Tolerance. Transplantation (2010) 90 (10):1043-7. doi: 10.1097/TP.0b013e3181efcfe9

3. O'Connell PJ, Zhang W, Menon MC, Yi Z, Schroppel B, Gallon L, et al. Biopsy Transcriptome Expression Profiling to Identify Kidney Transplants at Risk of Chronic Injury: A Multicentre, Prospective Study. Lancet (2016) 388 (10048):983-93. doi: 10.1016/S0140-6736(16)30826-1

4. Flechner SM, Kurian SM, Head SR, Sharp SM, Whisenant TC, Zhang J, et al. Kidney Transplant Rejection and Tissue Injury by Gene Profiling of Biopsies and Peripheral Blood Lymphocytes. Am J Transplant (2004) 4(9):1475-89. doi: 10.1111/j.1600-6143.2004.00526.x

5. Gu G, Huang Y, Wu C, Guo Z, Ma Y, Xia Q, et al. Differential Expression of Long Noncoding RNAs During Cardiac Allograft Rejection. Transplantation (2017) 101(1):83-91. doi: 10.1097/TP.0000000000001463

6. Tanay A, Regev A. Scaling Single-Cell Genomics From Phenomenology to Mechanism. Nature (2017) 541(7637):331-8. doi: 10.1038/nature21350

7. Han X, Wang R, Zhou Y, Fei L, Sun H, Lai S, et al. Mapping the Mouse Cell Atlas by Microwell-Seq. Cell (2018) 173(5):1307. doi: 10.1016/ j.cell.2018.05.012

8. Wu H, Malone AF, Donnelly EL, Kirita Y, Uchimura K, Ramakrishnan SM, et al. Single-Cell Transcriptomics of a Human Kidney Allograft Biopsy Specimen Defines a Diverse Inflammatory Response. J Am Soc Nephrol (2018) 29(8):2069-80. doi: 10.1681/ASN.2018020125

9. Wu C, Zhao Y, Xiao X, Fan Y, Kloc M, Liu W, et al. Graft-Infiltrating Macrophages Adopt an M2 Phenotype and Are Inhibited by Purinergic Receptor P2X7 Antagonist in Chronic Rejection. Am J Transplant (2016) 16 (9):2563-73. doi: 10.1111/ajt.13808

10. Chen S, Zhou Y, Chen Y, Gu J. Fastp: An Ultra-Fast All-in-One FASTQ Preprocessor. Bioinformatics (2018) 34(17):i884-90. doi: 10.1093/ bioinformatics/bty560

11. Ashburner M, Ball CA, Blake JA, Botstein D, Butler H, Cherry JM, et al. Gene Ontology: Tool for the Unification of Biology. Gene Ontol Consortium Nat Genet (2000) 25(1):25-9. doi: 10.1038/75556

12. Draghici S, Khatri P, Tarca AL, Amin K, Done A, Voichita C, et al. A Systems Biology Approach for Pathway Level Analysis. Genome Res (2007) 17 (10):1537-45. doi: 10.1101/gr.6202607

13. Yaari G, Bolen CR, Thakar J, Kleinstein SH. Quantitative Set Analysis for Gene Expression: A Method to Quantify Gene Set Differential Expression Including Gene-Gene Correlations. Nucleic Acids Res (2013) 41(18):e170. doi: 10.1093/nar/gkt660

14. Cao J, Spielmann M, Qiu X, Huang X, Ibrahim DM, Hill AJ, et al. The SingleCell Transcriptional Landscape of Mammalian Organogenesis. Nature (2019) 566(7745):496-502. doi: 10.1038/s41586-019-0969-x

15. Teniente-Serra A, Pizarro E, Quirant-Sánchez B, Fernández MA, Vives-Pi M, Martinez-Caceres EM. Identifying Changes in Peripheral Lymphocyte Subpopulations in Adult Onset Type 1 Diabetes. Front Immunol (2021) 12:784110. doi: 10.3389/fimmu.2021.784110

16. Girlanda R, Cheema AK, Kaur P, Kwon Y, Li A, Guerra J, et al. Metabolomics of Human Intestinal Transplant Rejection. Am J Transplant (2012) 12(Suppl 4):S18-26. doi: 10.1111/j.1600-6143.2012.04183.x

17. Heng B, Li Y, Shi L, Du X, Lai C, Cheng L, et al. A Meta-Analysis of the Significance of Granzyme B and Perforin in Noninvasive Diagnosis of Acute Rejection After Kidney Transplantation. Transplantation (2015) 99(7):147786. doi: $10.1097 /$ TP. 0000000000000567

18. Jindal R, Unadkat J, Zhang W, Zhang D, Ng TW, Wang Y, et al. Spontaneous Resolution of Acute Rejection and Tolerance Induction With IL-2 Fusion Protein in Vascularized Composite Allotransplantation. Am J Transplant (2015) 15(5):1231-40. doi: 10.1111/ajt.13118

19. Girlanda R, Kleiner DE, Duan Z, Ford EA, Wright EC, Mannon RB, et al. Monocyte Infiltration and Kidney Allograft Dysfunction During Acute Rejection. Am J Transplant (2008) 8(3):600-7. doi: 10.1111/j.16006143.2007.02109.x
20. Ensan S, Li A, Besla R, Degousee N, Cosme J, Roufaiel M, et al. Self-Renewing Resident Arterial Macrophages Arise From Embryonic CX3CR1(+) Precursors and Circulating Monocytes Immediately After Birth. Nat Immunol (2016) 17(2):159-68. doi: 10.1038/ni.3343

21. Beckers CML, Simpson KR, Griffin KJ, Brown JM, Cheah LT, Smith KA, et al. Cre/lox Studies Identify Resident Macrophages as the Major Source of Circulating Coagulation Factor XIII-A. Arterioscler Thromb Vasc Biol (2017) 37(8):1494-502. doi: 10.1161/ATVBAHA.117.309271

22. Zimmerman KA, Bentley MR, Lever JM, Li Z, Crossman DK, Song CJ, et al. Single-Cell RNA Sequencing Identifies Candidate Renal Resident Macrophage Gene Expression Signatures Across Species. J Am Soc Nephrol (2019) 30 (5):767-81. doi: 10.1681/ASN.2018090931

23. Schetters STT, Kruijssen LJW, Crommentuijn MHW, Kalay H, Ochando J, den Haan JMM, et al. Mouse DC-SIGN/CD209a as Target for Antigen Delivery and Adaptive Immunity. Front Immunol (2018) 9:990. doi: 10.3389/fimmu.2018.00990

24. Wilson KR, Villadangos JA, Mintern JD. Dendritic Cell Flt3 - Regulation, Roles and Repercussions for Immunotherapy. Immunol Cell Biol (2021) 99 (9):962-71. doi: 10.1111/imcb.12484

25. Mosser DM, Edwards JP. Exploring the Full Spectrum of Macrophage Activation. Nat Rev Immunol (2008) 8(12):958-69. doi: 10.1038/ nri2448

26. Kilberg MS, Shan J, Su N. ATF4-Dependent Transcription Mediates Signaling of Amino Acid Limitation. Trends Endocrinol Metab (2009) 20(9):436-43. doi: $10.1016 /$ j.tem.2009.05.008

27. Dai H, Lan P, Zhao D, Abou-Daya K, Liu W, Chen W, et al. PIRs Mediate Innate Myeloid Cell Memory to Nonself MHC Molecules. Science (2020) 368 (6495):1122-7. doi: 10.1126/science.aax4040

28. Reeve J, Einecke G, Mengel M, Sis B, Kayser N, Kaplan B, et al. Diagnosing Rejection in Renal Transplants: A Comparison of Molecular- and Histopathology-Based Approaches. Am J Transplant (2009) 9(8):1802-10. doi: 10.1111/j.1600-6143.2009.02694.x

29. Khatri P, Roedder S, Kimura N, De Vusser K, Morgan AA, Gong Y, et al. A Common Rejection Module (CRM) for Acute Rejection Across Multiple Organs Identifies Novel Therapeutics for Organ Transplantation. J Exp Med (2013) 210(11):2205-21. doi: 10.1084/jem.20122709

30. Roedder S, Sigdel T, Salomonis N, Hsieh S, Dai H, Bestard O, et al. The kSORT Assay to Detect Renal Transplant Patients at High Risk for Acute Rejection: Results of the Multicenter AART Study. PloS Med (2014) 11(11): e1001759. doi: 10.1371/journal.pmed.1001759

31. Zhao Q, Eichten A, Parveen A, Adler C, Huang Y, Wang W, et al. Single-Cell Transcriptome Analyses Reveal Endothelial Cell Heterogeneity in Tumors and Changes Following Antiangiogenic Treatment. Cancer Res (2018) 78 (9):2370-82. doi: 10.1158/0008-5472.CAN-17-2728

32. Farbehi N, Patrick R, Dorison A, Xaymardan M, Janbandhu V, Wystub-Lis K, et al. Single-Cell Expression Profiling Reveals Dynamic Flux of Cardiac Stromal, Vascular and Immune Cells in Health and Injury. Elife (2019) 8: e43882. doi: $10.7554 /$ eLife. 43882

33. Hipp MS, Kalveram B, Raasi S, Groettrup M, Schmidtke G. FAT10, a Ubiquitin-Independent Signal for Proteasomal Degradation. Mol Cell Biol (2005) 25(9):3483-91. doi: 10.1128/MCB.25.9.3483-3491.2005

34. Malik U, Javed A, Ali A, Asghar K. Structural and Functional Annotation of Human FAM26F: A Multifaceted Protein Having a Critical Role in the Immune System. Gene (2017) 597:66-75. doi: 10.1016/j.gene.2016.10.029

35. Lakkis FG, Li XC. Innate Allorecognition by Monocytic Cells and its Role in Graft Rejection. Am J Transplant (2018) 18(2):289-92. doi: 10.1111/ ajt.14436

36. Oberbarnscheidt MH, Zeng Q, Li Q, Dai H, Williams AL, Shlomchik WD, et al. Non-Self Recognition by Monocytes Initiates Allograft Rejection. J Clin Invest (2014) 124(8):3579-89. doi: 10.1172/JCI74370

37. Niethamer TK, Stabler CT, Leach JP, Zepp JA, Morley MP, Babu A, et al. Defining the Role of Pulmonary Endothelial Cell Heterogeneity in the Response to Acute Lung Injury. Elife (2020) 9:e53072. doi: 10.7554/ eLife. 53072

38. Jambusaria A, Hong Z, Zhang L, Srivastava S, Jana A, Toth PT, et al. Endothelial Heterogeneity Across Distinct Vascular Beds During Homeostasis and Inflammation. Elife (2020) 9:e51413. doi: 10.7554/eLife.51413 
39. Abrahimi P, Qin L, Chang WG, Bothwell AL, Tellides G, Saltzman WM, et al. Blocking MHC Class II on Human Endothelium Mitigates Acute Rejection. JCI Insight (2016) 1(1):e85293. doi: 10.1172/jci.insight.85293

40. Merola J, Reschke M, Pierce RW, Qin L, Spindler S, Baltazar T, et al. Progenitor-Derived Human Endothelial Cells Evade Alloimmunity by CRISPR/Cas9-Mediated Complete Ablation of MHC Expression. JCI Insight (2019) 4(20):e129739. doi: 10.1172/jci.insight.129739

41. Bates EE, Ravel O, Dieu MC, Ho S, Guret C, Bridon JM, et al. Identification and Analysis of a Novel Member of the Ubiquitin Family Expressed in Dendritic Cells and Mature B Cells. Eur J Immunol (1997) 27(10):2471-7. doi: $10.1002 /$ eji. 1830271002

42. Choi JW, Kim YH, Oh JW. Comparative Analyses of Signature Genes in Acute Rejection and Operational Tolerance. Immune Netw (2017) 17(4):237-49. doi: 10.4110/in.2017.17.4.237

43. Hruba P, Madill-Thomsen K, Mackova M, Klema J, Maluskova J, Voska L, et al. Molecular Patterns of Isolated Tubulitis Differ From Tubulitis With Interstitial Inflammation in Early Indication Biopsies of Kidney Allografts. Sci Rep (2020) 10(1):22220. doi: 10.1038/s41598-020-79332-9
Conflict of Interest: The authors declare that the research was conducted in the absence of any commercial or financial relationships that could be construed as a potential conflict of interest.

Publisher's Note: All claims expressed in this article are solely those of the authors and do not necessarily represent those of their affiliated organizations, or those of the publisher, the editors and the reviewers. Any product that may be evaluated in this article, or claim that may be made by its manufacturer, is not guaranteed or endorsed by the publisher.

Copyright $\odot 2022$ Tang, Wang, Zhang, Li, Chen, Gao, Dai, Lin, He, Wu and Shi. This is an open-access article distributed under the terms of the Creative Commons Attribution License (CC BY). The use, distribution or reproduction in other forums is permitted, provided the original author(s) and the copyright owner(s) are credited and that the original publication in this journal is cited, in accordance with accepted academic practice. No use, distribution or reproduction is permitted which does not comply with these terms. 